\title{
Utility of presepsin, soluble triggering receptor expressed on myeloid cells-I, and neutrophil CD64 for early detection of neonatal sepsis
}

This article was published in the following Dove Medical Press journal: Infection and Drug Resistance

\author{
Asmaa A El-Madbouly' \\ Asmaa A El Sehemawy ${ }^{2}$ \\ Noha A Eldesoky ${ }^{3}$ \\ Heba Mohammed Abd \\ Elgalil ${ }^{4}$ \\ Amal M Ahmed ${ }^{4}$ \\ 'Clinical Pathology Department, \\ Faculty of Medicine, Al-Azhar \\ University, Cairo, Egypt; ${ }^{2}$ Pediatrics \\ Department, Faculty of Medicine, \\ Al-Azhar University, Cairo, Egypt; \\ ${ }^{3}$ Biochemistry Department, Faculty of \\ Pharmacy, Al-Azhar University, Cairo, \\ Egypt; ${ }^{4}$ Community and Occupational \\ Medicine Department, Faculty of \\ Medicine, Al-Azhar University, Cairo, \\ Egypt
}

Background: Neonatal sepsis (NS) is an important cause of morbidity and mortality among newborns. Its diagnosis depends mainly on blood culture that takes at least 48 hours to give results. Therefore, searching for biomarkers for early diagnosis is of value. We aimed to assess presepsin, soluble triggering receptor expressed on myeloid cells (sTREM-1), and neutrophil CD64 (nCD64) as early diagnostic biomarkers in NS, and to compare them individually and in combination.

Methods: This hospital-based case-control study has been conducted on 60 full-term neonates recruited from the neonatal intensive care unit, Al-Zahraa Hospital, Al-Azhar University, Cairo, Egypt. Thirty infants with sepsis were compared to 30 postnatal age- and sex-matched healthy controls. Studied neonates were evaluated using clinical and laboratory indicators for sepsis. nCD64 was measured by flow cytometry and, serum presepsin and sTREM-1 were measured by ELISA.

Results: Presepsin, sTREM-1, and nCD64 levels were significantly elevated in septic neonates vs control group $(P<0.05)$. The sensitivities of presepsin, sTREM, and nCD64 were $100 \%, 96.7 \%$, and $86.7 \%$, respectively. Presepsin had the best diagnostic performance in early diagnosis of NS followed by sTREM-1 and nCD64.

Conclusion: Presepsin and sTREM-1 are promising biomarkers in screening for NS in comparison with nCD64. However, nCD64 is better used in combination with other biomarkers as CRP. Keywords: neonatal sepsis, presepsin, sTREM-1, nCD64, diagnosis, screening

\section{Introduction}

Neonatal sepsis (NS) is a clinical syndrome occurring in infants $\leq 28$ days of life, characterized by symptoms and signs of infection with or without accompanying bacteremia. $^{1}$

Sepsis is a global health problem as it is an important cause of morbidity and mortality among newborn infants. The overall incidence of NS ranges from 1 to 5 cases per 1,000 live births in developed countries, compared to 49-170 per 1,000 live births in some population-based studies in the developing countries, with case fatality rates ranging from $2 \%$ to $60 \%$. $^{2,3}$

In Egypt, around $80 \%$ of early childhood deaths take place before a child's first birthday, with slightly more than half (52\%) occurring during the first month of life. The neonatal mortality rates were 14 deaths per 1,000 births. $^{4}$

Early diagnosis of sepsis is a clinical challenge as the clinical symptoms of NS are subtle, late, and nonspecific. Blood culture is still the gold standard for the diagnosis of sepsis, but minimum 48 hours is required for the earliest result and it can be negative
Elgalil

Community and Occupational Medicine Department, Faculty of Medicine,

Al-Azhar University, Yousef Abbas Street,

Cairo, I I754, Egypt

Tel +20 II 534I I 236

Fax +20223868126

Email bashandy@azhar.edu.eg 
in spite of clinical signs of sepsis. Delaying the treatment of a bacteremic infant leads to an increase in mortality while starting antibiotic therapy to every infant in the presence of clinical suspicion causes antibiotic overuse and resistance in addition to nosocomial infections because of unnecessary hospitalization as well as separation from parents. Thus, there is an urgent need for early diagnostic accurate markers of sepsis so that timely and appropriate use of antibiotics can be initiated leading to better outcomes. ${ }^{5}$

Neutrophil cluster of differentiation 64 (nCD64) is a high-affinity biomarker that binds to immunoglobulin G. It is normally expressed on monocytes but in cases of infection or inflammation, CD64 is normally expressed on monocytes but in cases of infection or inflammation, it is upregulated rapidly on neutrophils under stimulation by inflammatory cytokines. ${ }^{6}$

Quantitative measurement of nCD64 expression has been suggested as a diagnostic biomarker of NS. ${ }^{7}$

Among the promising biomarkers is the presepsin (sCD14-ST). It is a specific, high-affinity receptor for complexes of lipopolysaccharides and is involved in the recognition of a wide variety of bacterial products, such as peptidoglycans, the major cell-wall component of Grampositive bacteria. Following its stimulation by pathogens, a soluble CD14 subtype called presepsin is released by shedding from the surface of the membranes of various immune cells, such as macrophages, monocytes, and neutrophils. ${ }^{8}$

The triggering receptor expressed on myeloid cells-1 (TREM-1) is a glycoprotein member of the immunoglobulin superfamily that mediates the acute inflammatory response to microbial products. TREM-1 is shed from the membrane of activated phagocytes and can be detected in a soluble form in serum and body fluids (sTREM-1). ${ }^{9}$ sTREM-1 levels are not detectable at baseline in normal individuals. ${ }^{10}$ It is an important signaling receptor that magnifies the inflammatory reaction by elevating pro-inflammatory cytokines and blocking interleukin (IL)-10 syntheses. ${ }^{11}$

The aim of this study was to investigate and compare the utility of nCD64, presepsin, and sTREM-1, as early diagnostic biomarkers of NS individually and in combination to determine the most useful marker(s); hence, proper timely treatment could be initiated to reduce the consequences of sepsis.

\section{Materials and methods} Study design

The current study was a prospective hospital-based case-control study carried out during the period from June 2017 to March 2018 at Al-Zahraa University Hospital (Cairo, Egypt).

\section{Study population}

A total of 60 full-term neonates according to New Ballard score were recruited in this study. ${ }^{12}$ Thirty of them were considered clinically to have sepsis (case group) on the basis of sepsis score reported by hematological scoring system (HSS) and modified clinical sepsis score by Töllner. ${ }^{13}$ The threshold for HSS and Tollnar scores were $\geq 3$ and $\geq 10$, respectively. ${ }^{13}$ They were admitted to neonatal intensive care unit (NICU) at Al-Zahraa University Hospital and subdivided according to the onset of developing sepsis into early onset sepsis (EOS) (17 neonates) and late onset sepsis (LOS) (13 neonates) groups.

A group of 30 postnatal age- and sex-matched newborns, with normal physical examination and no clinical findings of sepsis were chosen as control group. They were selected from the outpatient neonatology clinic coming for follow-up.

Neonates with major congenital malformations, chromosomal abnormalities, and fetal hydrops were excluded from the study.

\section{Sample size calculation}

Sample size was calculated using EPI Info ${ }^{\mathrm{TM}}$ version 7 (US Centers for Disease Control and Prevention, Atlanta, GA, USA) according to the annual flow at the hospital and prevalence of NS (3.2\%) as there was wide variability of infection rates in the NICUs ranged from 3.2 to 30 per 100 admissions or discharges using a $5 \%$ margin of error, confidence level of $95 \%$, and response distribution of $80 \%{ }^{14}$

Written informed consent was obtained from the parents of each neonate. The study protocol was approved by the local Ethics Committee of AL-Azhar University, Faculty of Medicine (for Girls), and all procedures were in accordance with the Helsinki Declaration.

\section{All neonates in the present study were subjected to the following Detailed medical history and thorough clinical examination}

\section{Laboratory investigations}

I. Complete blood count (KX-21N; Sysmex, Kobe, Japan) and peripheral blood smear for differential count.

II. Alanin transaminase, aspartate transaminase, blood urea, and serum creatinine (Cobas Integra 400 plus; Hoffman-La Roche Ltd., Basel, Switzerland).

III. Arterial blood gases (Cobas 221; Hoffman-La Roche Ltd.).

IV. Semi quantitative CRP measurement by latex agglutination (Omega Diagnostics Ltd, Alva, UK) 
V. Blood culture: $2 \mathrm{~mL}$ of blood was injected into the Bact/ Alert culture bottle under complete aseptic conditions. The inoculated culture bottles were placed in the Bact/ Alert instrument (bio-Mérieux, Marcy l'Etoile, France) as soon as possible, for incubation and monitoring. Positive samples were Gram stained and subcultured on blood agar, MacConkey agar, and sabouraud dextrose agar supplemented with chloramphenicol (Oxoid, England) and incubated in appropriate temperature. Full identification of organisms was done with Vitek 2 compact (bio-Mérieux). Coagulase-negative Staphylococcus was identified as a causative pathogen for sepsis by its isolation from two positive blood cultures.

VI. Determination of surface nCD64 by flow cytometry: flow cytometry was performed on EDTA peripheral blood specimens obtained before the administration of any treatment and analyzed within 24 hours of collection.

It was conducted using four colors FACS Calibur (BD Biosciences, San Jose, CA, USA). Cell Quest Pro software (BD Biosciences) was used for data analysis. Compensation setting was established before acquiring the samples using color calibrite beads (lot no. 5093879, BD Biosciences). After adjusting the sample count for acquisition, unstained samples were acquired to detect the sample autofluorescence. Isotype controls, mouse IgG1 fluorescein isothiocyanate (FITC) control, and mouse IgG PerCP control were obtained from BD Biosciences for the detection of nonspecific binding.

For each sample, $50 \mu \mathrm{L}$ of EDTA blood was stained by FITC-conjugated anti-human CD64 (Immunotech, lot no. 44, cat. no. IM1604U; Beckman Coulter, Marsellia, France) and peridin chlorophyll protein complex
(PerCP)-conjugated anti-human CD45 (lot no. 6039924 clone (2D1), cat. no. 345809, BD Biosciences). The optimal concentration was determined for each antibody by titration experiment.

After 15 minutes of incubation in the dark, each sample was washed, centrifuged, and lysed using BD FACS LYSE (cat. no. 550811, lot no. 5329747; BD Biosciences). Then the samples were washed and resuspended in $200 \mu$ FACS buffer (cat. no. 550811, lot no. 5329747; BD Biosciences).

VII. Gating strategy: using CD45 and side scatter (CD45/ $\mathrm{SS}$ ), initial gating was performed on neutrophil area in the dot plot graph (Figure 1A). Data were expressed as mean fluorescence intensity of nCD64 using single histogram (Figure 1B).

VII. Measurement of serum levels of presepsin and sTREM-1: the serum for measurement of presepsin and sTREM-1 was kept at $-20^{\circ} \mathrm{C}$ until analysis. Serum presepsin and sTREM-1 were measured by ELISA (cat. no. 11141; Glory Science Co., Ltd, Hong Kong, China and cat. no. E0310Hu, Biotech Co., Ltd, Shanghai, China) respectively, with a detection range of 8-300 $\mathrm{pg} / \mathrm{mL}$ for presepsin and 3-900 ng/L for sTREM-1.

All laboratory investigations were performed at Clinical Pathology Department, AL-Zahraa University Hospital, AL-Azhar University.

\section{Statistical design}

Continuous data were described in terms of mean $\pm \mathrm{SD}$, whereas categorical variables were described in number and percentage. Chi-squared test was used for the comparison of categorical variables while Student's $t$-test was used to compare between quantitative data. Correlation was used to explore the
A

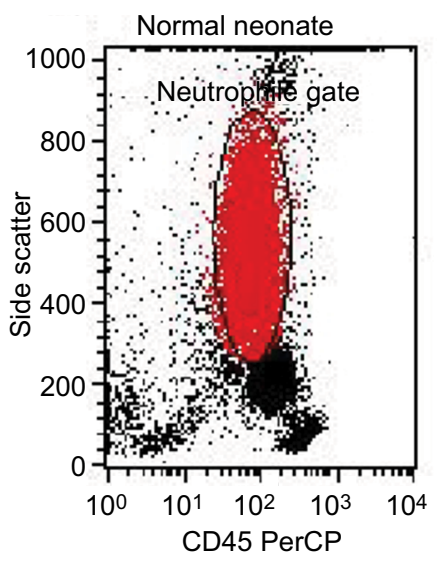

B

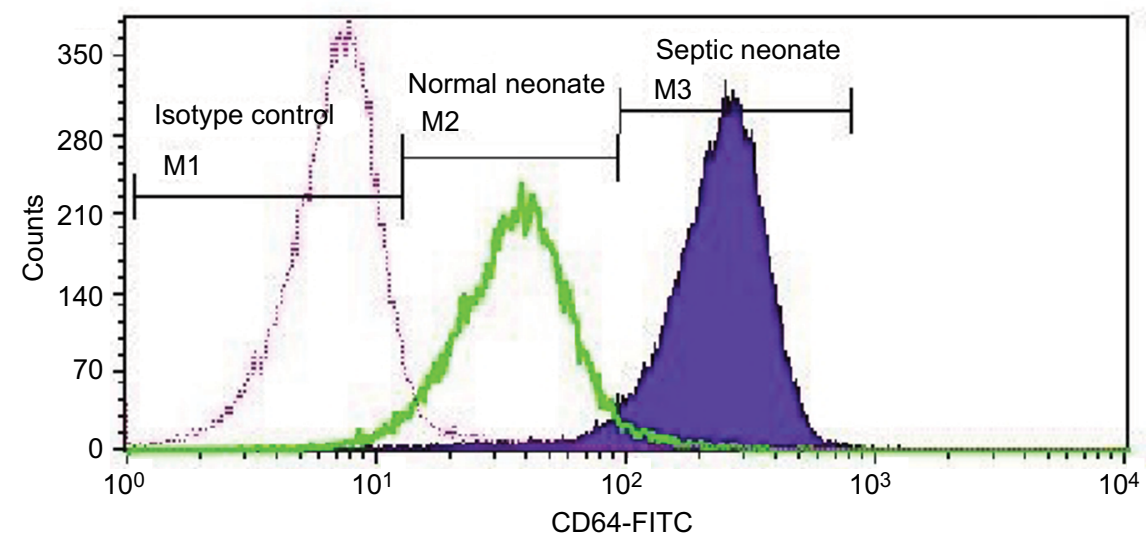

Figure I (A) Neutrophile gating by CD45/SS. (B) MFI of nCD64 for an isotype control (MI), normal neonate (M2), and septic neonate (M3). Abbreviations: FITC, fluorescein isothiocyanate; MFI, mean fluorescence intensity; PerCP: peridinin chlorophyll protein complex. 
relationship between quantitative variables. Receiver operating characteristic (ROC) curve was used to assess the best cut-off point with sensitivity, specificity, positive predictive value (PPV) and negative predictive value (NPV). Significance level was taken at $P$-value $\leq 0.05$. All analyses were performed using SPSS version 16 (SPSS Inc., Chicago, IL, USA).

\section{Results}

Sixty full-term neonates were included in the study. Thirty of them with clinically suspected sepsis (sepsis group) were compared to 30 postnatal age- and sex-matched healthy neonates (control group). Within sepsis group, $56.7 \%$ of neonates had EOS as they developed sepsis within the first 72 hours after birth and $43.3 \%$ developed LOS after 72 hours of birth.

The general, clinical, and routine laboratory data of both the studied groups are presented in Table 1.
Neonates of septic group had significant lower mean of gestational age, length, and platelet count than controls. While, they had significant higher mean of total leucocytic count (WBCs) vs control $(P<0.05)$.

When compared between EOS and LOS infants, no statistical significant difference was found in terms of demographic, clinical, and laboratory data $(P>0.05)$ except for platelet count that was lower in EOS than LOS $(P<0.05)$ (Table S1).

Blood culture was positive in $90.0 \%$ of all septic neonates (Figure 2A). Klebsiella pneumonia was the most isolated causative organism of sepsis (44.4\%) followed by coagulasenegative Staphylococci and Acinetobacter (26.0\% and 22.2\%, respectively) (Figure $2 \mathrm{~B}$ ).

CRP, presepsin, nCD64, and sTREM-1 levels were significantly elevated in septic neonates vs control group $(P<0.05)$ (Table 2).

Table I General, clinical, and laboratory data of studied neonates

\begin{tabular}{|c|c|c|c|c|}
\hline \multirow[t]{2}{*}{ Items } & \multicolumn{2}{|l|}{ Studied groups } & \multirow[t]{2}{*}{ Test of significance } & \multirow[t]{2}{*}{$P$-value } \\
\hline & $\begin{array}{l}\text { aSepsis group, } n=30 \text {, } \\
n(\%),(\text { mean } \pm \text { SD) }\end{array}$ & $\begin{array}{l}\text { Control group, } n=30 \\
n(\%),(\text { mean } \pm \text { SD) }\end{array}$ & & \\
\hline \multicolumn{5}{|l|}{ Sex } \\
\hline Male & $20(66.6 \%)$ & $18(60.0 \%)$ & $\chi^{2}=0.8$ & 0.7 \\
\hline Female & $10(33.4 \%)$ & $12(40.0 \%)$ & & \\
\hline Gestational age (weeks) & $37.9 \pm 1.7$ & $39 \pm 1.4$ & $t$-test $=2.4$ & $0.02^{\mathrm{b}}$ \\
\hline Birth weight (g) & $2.9 \pm 0.7$ & $3 \pm 0.3$ & $t$-test $=0.5$ & 0.7 \\
\hline Length $(\mathrm{cm})$ & $47.9 \pm 2$ & $49.1 \pm 1.5$ & $t$-test $=2.1$ & $0.04^{\mathrm{b}}$ \\
\hline Head circumference $(\mathrm{cm})$ & $31.6 \pm 8$ & $34 \pm 0.9$ & $t$-test $=1.2$ & 0.2 \\
\hline WBCs $\left(10^{3} / \mu \mathrm{L}\right)$ & $19.7 \pm 8.3$ & $11 \pm 3.6$ & $t$-test $=4$ & $0.00 \mathrm{I}^{\mathrm{b}}$ \\
\hline $\mathrm{Hb}(\mathrm{g} / \mathrm{dL})$ & $12.3 \pm 2.1$ & $13.7 \pm 1.7$ & $t$-test $=1.6$ & 0.1 \\
\hline HCT (\%) & $38.2 \pm 6.3$ & $37.5 \pm 8.4$ & $t$-test $=0.3$ & 0.8 \\
\hline Platelets $\left(10^{3} / \mu \mathrm{L}\right)$ & $142 \pm 82$ & $240 \pm 74$ & $t$-test $=3.8$ & $0.000^{\mathrm{b}}$ \\
\hline Apgar score at 5 minutes & $8 \pm 0.8$ & - & - & - \\
\hline Tollner score & $12.7 \pm 3.3$ & - & - & - \\
\hline Hematological score & $4.8 \pm 0.7$ & - & - & - \\
\hline
\end{tabular}

Notes: "Comparison was done between EOS and LOS groups using Student's independent $t$-test in all quantitative variables. ${ }^{\circ} \leq 0.05$ is significant. “-” indicates apgar score was not done for control group.

Abbreviations: EOS, early onset sepsis; Hb, hemoglobin; HCT, hematocrit; LOS, late onset sepsis; WBC, white blood cell.

A

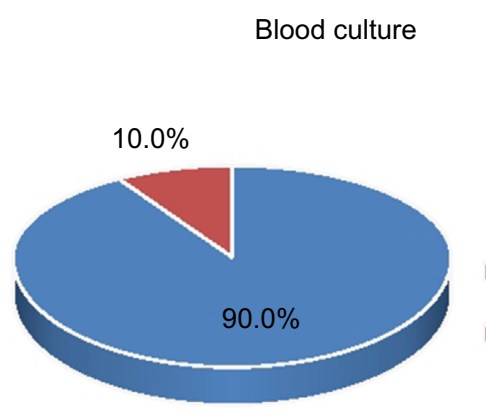

B

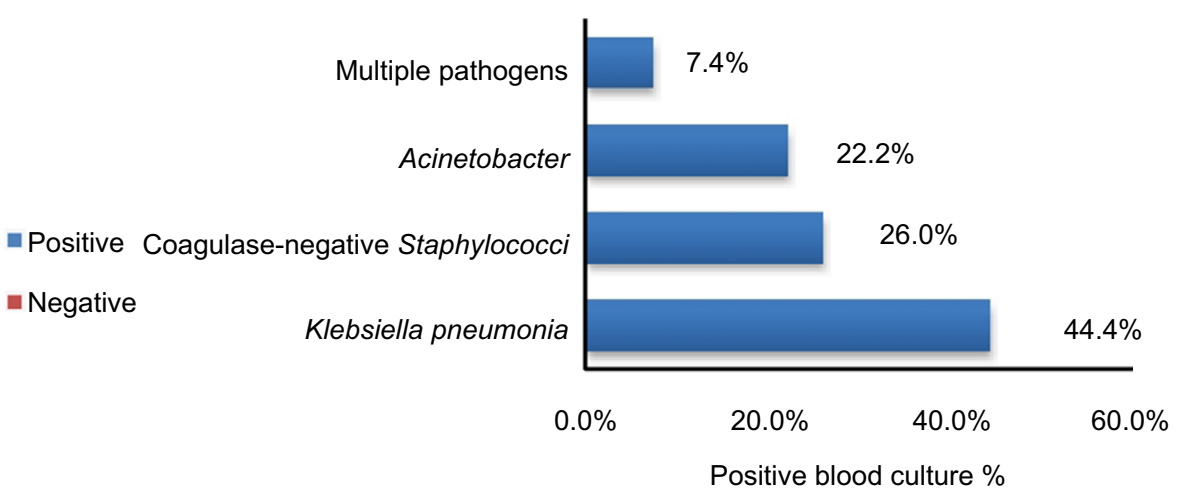

Figure 2 (A) Blood culture results among septic neonates. (B) Microorganisms identified in blood culture of septic neonates. 
No significant differences was detected in in CRP, presepsin, nCD64, and sTREM levels between EOS and LOS groups $(P>0.05)$. There was a significant difference between each group vs control group (Table S2).

According to blood culture results, the sepsis group was subdivided into proven sepsis (with positive blood culture) and probable sepsis (with negative blood culture). The levels of CRP, presepsin, nCD64, and sTREM-1 in both the groups showed significant difference for CRP only, while there was significant difference between the levels of all biomarkers vs control group separately (Table S3).

Sensitivity, specificity, PPV, and NPV for each marker, and their combinations are summarized in Table 3, using optimal cut-off values.

ROC curve for CRP, prespsin, sTREM-1, nCD64, and combined (prespsin, sTREM-1, and nCD64) for early detection of NS (Figure 3A).

Figure 3B shows the ROC curve for combined (CRP, nCD64), (CRP, sTREM-1), and (CRP, sTREM-1, nCD64).
There were significant positive correlations between CRP with either sTREM-1 or nCD64 and also between sTREM-1 and nCD64 (Table 4).

\section{Discussion}

Sepsis is the second most frequent direct cause of death among neonates. It is obvious that early diagnosis and timely treatment of NS are vital to prevent severe and lifethreatening complications and decrease the emergence of antibiotic resistance and financial burden for family and society. ${ }^{15}$

This study included 30 clinically septic neonates and 30 control neonates. Within sepsis group, EOS was higher (56.7\%) than LOS (43.3\%). This observation was constant with previous studies. ${ }^{16,17}$

Blood culture is considered the gold standard for the diagnosis of NS. In the current study, $10 \%$ of neonates with clinically suspected sepsis had negative blood cultures. This was in accordance with a study done by $\mathrm{Ng}$ and Lam, who

Table 2 Levels of CRP, presepsin, nCD64, and sTREM-I in studied groups

\begin{tabular}{|c|c|c|c|c|}
\hline \multirow[t]{2}{*}{ Items } & \multicolumn{2}{|l|}{ Studied groups } & \multirow[t]{2}{*}{$t$-test } & \multirow[t]{2}{*}{$P$-value } \\
\hline & aSepsis group, $n=30$ & Control group, $n=30$ & & \\
\hline \multicolumn{5}{|l|}{$\mathrm{CRP}(\mathrm{mg} / \mathrm{L})$} \\
\hline Range & $6-60$ & $6-12$ & 3.8 & $0.00 I^{\mathrm{b}}$ \\
\hline Mean \pm SD & $28.6 \pm 17$ & $10.8 \pm 6$ & & \\
\hline \multicolumn{5}{|c|}{ Presepsin $(\mathrm{pg} / \mathrm{mL})$} \\
\hline Range & II $74-4854$ & 168.9-935.6 & 9.3 & $0.00 I^{\mathrm{b}}$ \\
\hline Mean \pm SD & $3394 \pm 1,152$ & $560.6 \pm 221$ & & \\
\hline \multicolumn{5}{|l|}{ nCD64 (MFI) } \\
\hline Range & $29-1647$ & $16.5-95.5$ & 2.8 & $0.007^{b}$ \\
\hline Mean \pm SD & $380 \pm 69.5$ & $45.9 \pm 22.7$ & & \\
\hline \multicolumn{5}{|l|}{ sTREM-I (ng/L) } \\
\hline Range & $28.7-335$ & $27.4-66$ & 3.7 & $0.00 I^{\mathrm{b}}$ \\
\hline Mean \pm SD & $105 \pm 65$ & $43.3 \pm 12.2$ & & \\
\hline
\end{tabular}

Notes: ${ }^{2}$ Comparison was done between EOS and LOS groups using Student's independent $t$-test in all quantitative variables. ${ }^{\mathrm{b}} \leq 0.05$ is significant.

Abbreviations: MFI, mean fluorescence intensity; nCD64, neutrophil CD64; sTREM-I, soluble triggering receptor expressed on myeloid cells-I.

Table 3 Cut-off values, sensitivities, and specificities for early detection of neonatal sepsis as calculated on ROC curves for studied biomarkers

\begin{tabular}{|l|l|l|l|l|}
\hline Variables (cut-off values) & Sensitivity (\%) & Specificity (\%) & PPV (\%) & NPV (\%) \\
\hline Presepsin $(\mathrm{pg} / \mathrm{mL}) \geq 767$ & 100 & 86.7 & 84.4 & 100 \\
\hline sTREM-I (ng/L) $\geq 69.8$ & 96.7 & 86.7 & 83.9 & 92.9 \\
\hline nCD64 (MFI) $\geq 44.3$ & 86.7 & 73.3 & 72.0 & 69.2 \\
\hline Presepsin + sTREM-I+ nCD64 & 96.3 & 77.8 & 84.4 & 100.0 \\
\hline CRP $(\mathrm{mg} / \mathrm{L}) \geq 6$ & 85.2 & 39.0 & 67.6 & 64.0 \\
\hline CRP, nCD64 & 96.3 & 22.2 & 65 & 80 \\
\hline CRP, sTREM-I & 96.3 & 39 & 71 & 87.5 \\
\hline CRP, sTREM-I, nCD64 & 100 & 77 & 64.3 & 100 \\
\hline
\end{tabular}

Abbreviations: ROC, receiver operating characteristic; MFI, mean fluorescence intensity; PPV, positive predictive value; NPV, negative predictive value; nCD64, neutrophil CD64; sTREM-I, soluble triggering receptor expressed on myeloid cells-I. 
A

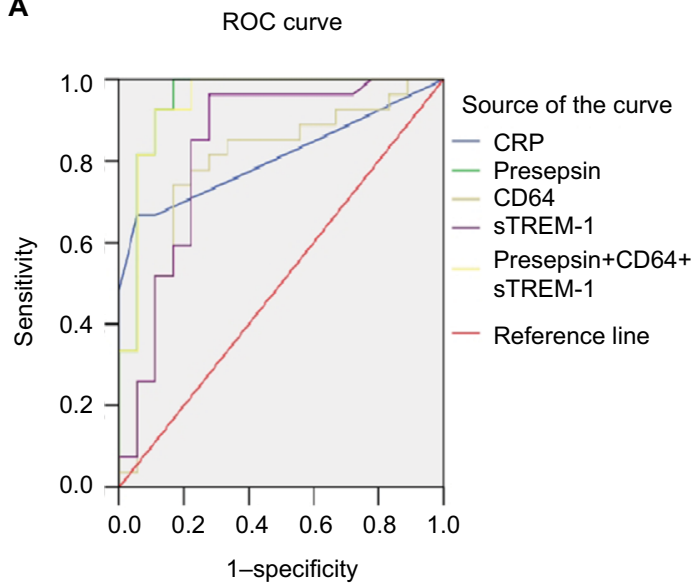

B

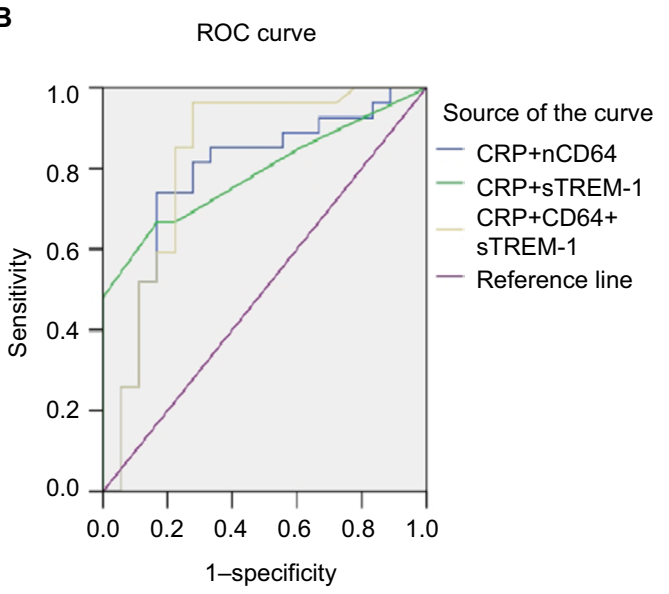

Figure 3 (A) ROC curve for CRP, prespsin, sTREM-I, nCD64, separately and in combination (prespsin, sTREM-I, and nCD64). (B) ROC curve for combined CRP and nCD64, CRP and sTREM-I, and CRP, sTREM-I, and nCD64.

Abbreviations: ROC, receiver operating characteristic; nCD64, neutrophil CD64; sTREM-I, soluble triggering receptor expressed on myeloid cells-I.

Table 4 Correlations between CRP, sTREM-I, and nCD64

\begin{tabular}{|l|l|l|}
\hline Items & $\boldsymbol{r}$ & $\boldsymbol{P}$-value \\
\hline CRP with sTREM-I & 0.534 & $0.024^{\mathrm{a}}$ \\
\hline CRP with nCD64 & $0.43 \mathrm{I}$ & $0.042^{\mathrm{a}}$ \\
\hline sTREM-I with nCD64 & 0.504 & $0.04 \mathrm{I}^{\mathrm{a}}$ \\
\hline
\end{tabular}

Note: ${ }^{a} P \leq 0.05$ is significant.

Abbreviations: nCD64, neutrophil CD64; sTREM-I, soluble triggering receptor expressed on myeloid cells-I.

reported that blood culture sensitivity in NS varies from $11 \%$ to $78 \% .{ }^{18}$ Also, Du et al reported that out of the 88 suspected septic neonates, pathogenic organisms were isolated from only $23 \%$ of their blood cultures. ${ }^{19}$

In the present study, K. pneumonia was the most frequent isolated organism from blood of neonates with sepsis (44.4\%) followed by coagulase-negative Staphylococci $(26 \%)$. This was in agreement with the studies of De Benedetti et al and Dzwonek et al, in which nearly half of the positive blood cultures grew $K$. pneumonia. ${ }^{20,21}$ On the contrary, Osman et al demonstrated that the most common isolated organism from septic neonates was coagulase-negative Staphylococci (17.5\%) followed by Staphylococcus aureus (12.5\%) and K. pneumonia $(10 \%))^{22}$ This difference may be attributed to variation in local epidemiology and the microbial etiology of sepsis in addition to different care practices between medical centers.

In the current study, the presepsin level was significantly higher in sepsis group than control group $(P<0.05)$. AUC for presepsin was higher (0.95) compared to CRP (0.79). The cut-off value at $767 \mathrm{pg} / \mathrm{mL}$ showed a sensitivity of $100 \%$ and specificity of $86.7 \%$. The PPV and NPV were $84.4 \%$ and $100 \%$, respectively, while the comparable figures for CRP were lower $(85.2 \%, 39 \%, 67.6 \%, 64 \%$, respectively), suggesting the potential utility of presepsin as a reliable marker for early diagnosis of NS.

These findings were in accordance with Małgorzata et al who demonstrated that the mean value of presepsin in septic newborn was significantly higher than control. ${ }^{23}$ Similarly, in 2016, Topcuoglu et al found significant higher presepsin levels in preterm infants with LOS vs controls. ${ }^{24}$ Moreover, they claimed that presepsin could be used as a useful marker to monitor treatment response, as its levels decrease over time with treatment.

However, due to the lack of information about presepsin reference range in neonates, many studies reported different cut-off values. ${ }^{22,25,26}$ Relatively different cut-off values may be due to the use of different measurement methods.

The current study also evaluated STREM-1 as a diagnostic biomarker for NS. The mean level of sTREM-1 was significantly higher in sepsis group than control group. ROC curve analysis revealed that the cut-off value of sTREM-1 at 69.8 ng/L had $96.7 \%$ sensitivity and $86.7 \%$ specificity. The PPV and NPV were $83.9 \%$ and $92.9 \%$, respectively.

These findings were consistent with a previous study by Adly et al, who reported that elevated sTREM-1 could be considered an early marker for NS and at a cut-off value 310 $\mathrm{pg} / \mathrm{mL}$, it has $100 \%$ sensitivity and specificity; furthermore, it reflected sepsis severity and poor prognosis. ${ }^{27}$

Similarly, Saldir et al revealed that the levels of sTREM-1 in serum of neonates with LOS increased significantly, suggesting that sTREM-1 had a considerable diagnostic performance in LOS and in monitoring the response to therapy thereafter. ${ }^{28}$ 
Neutrophil surface CD64 expression has shown particular promise as an early marker for sepsis. Technically, measurement of nCD64 by flow cytometry is rapid and needs a small blood volume. ${ }^{29}$ Moreover, the expression of nCD64 is not affected by transient tachypnea of the newborn, respiratory distress syndrome and other non-infective perinatal events. ${ }^{30}$

In the present study, nCD64 was significantly higher in infected neonates than control group $(P<0.05)$; and AUC was 0.78 with sensitivity and specificity of $86.7 \%$ and $73.3 \%$, respectively. The PPV and NPV were $72 \%$ and $69.2 \%$, respectively, with best cut-off value $\geq 44.3 \mathrm{MFI}$.

Our results were in concordance with Morsy et al who revealed in an Egyptian study that nCD64 increased significantly in sepsis group compared to the control group with a sensitivity of $95 \% .{ }^{31}$ Similarly, other studies reported increased expression of nCD64 in neonates with sepsis..$^{32,33}$

A meta-analysis by Jia et al also concluded that nCD64 expression can be used as an additional test in the diagnosis of neonatal infection. ${ }^{34}$

Different cut-off values for nCD64 were used in several studies with different sensitivities and specificities. ${ }^{17,29,30}$ These differences might be explained in several ways, such as the differences of the studied populations or the technical differences in the measurement methodology among different studies.

In our study, upon comparing the diagnostic performances of the studied three markers, presepsin had the best performance followed by sTREM-1 and lastly nCD64.

Similarly, nCD64 showed a lower diagnostic performance compared with presepsin in many clinical studies. ${ }^{8,35,36}$ Also, the results of a meta-analysis by Shi et al indicated that the nCD64 should not be used as a diagnostic marker alone for $\mathrm{NS}^{37}$

In comparison with nCD64, sTREM-1 in the present work had better performance. On the contrary, the study by Mazzucchelli et al reported that sTREM-1 showed less diagnostic accuracy than nCD64; however, that study was performed on preterm neonates with LOS. ${ }^{38}$

In the present study, AUC for the combination of the three markers was 0.94 ; sensitivity, specificity, PPV, and NPV were $96.3 \%, 77.8 \%, 84.4 \%, 100.0 \%$, respectively, at the same cut-off values, which could add a value to the diagnostic performance of nCD64 as it increased both the sensitivity and specificity. But for presepsin and STREM-1, the combination decreases both the sensitivity and specificity.

Combinations of different biomarkers may improve their sensitivity and/or specificity. Individually, they may not possess high sensitivity or specificity and are generally more helpful when considered together because sepsis is a complex and dynamic syndrome. ${ }^{29}$

In the present study, the concomitant measurement of nCD64 with CRP alone or even with sTREM-1 improved the sensitivity (96.3\% and $100 \%$, respectively), but it was at the expense of specificity (22.2\% and $77 \%$, respectively). Significant positive correlations between both the biomarkers nCD64 and sTREM-1 and CRP were also observed. These results clarified that this combination could be helpful in guiding decisions to start empirical antibiotic therapy but not in taking decisions to withhold antibiotic therapy.

These results were in accordance with other studies by Sabryetal and Sarode et al. ${ }^{39,40}$ However, Morsy et al found no significant correlation between CRP and CD64 in cases with NS. ${ }^{31}$

\section{Conclusion}

Presepsin, sTREM-1, and nCD64 levels were significantly higher in septic neonates than healthy controls, suggesting their potential use for screening and early diagnosis of NS in routine clinical situations. Either presepsin or sTREM-1 was a superior diagnostic marker for the early detection of NS; they can be used individually, while nCD64 should be combined with other markers (as CRP and hematological markers) that enhance its diagnostic accuracy for NS. However, the availability of daily testing, cost, and turnaround time of biomarkers in clinical laboratories would be somewhat dependent on the institutional resources. Future studies on larger scale should be applied.

\section{Disclosure}

The authors report no conflicts of interest in this work.

\section{References}

1. Ayub A, Chishti AL, Hassen KA. The validity of hematologic markers for diagnosis of neonatal sepsis. Ann King Edw Med Univ. 2015;21(4):240.

2. Thaver D, Zaidi AK. Burden of neonatal infections in developing countries: a review of evidence from community-based studies. Pediatr Infect Dis J. 2009;28(1 Suppl):S3-S9.

3. Bulkowstein S, Ben-Shimol S, Givon-Lavi N, Melamed R, Shany E, Greenberg D. Comparison of early onset sepsis and community-acquired late onset sepsis in infants less than 3 months of age. BMC Pediatr. 2016;16(1):82.

4. El-Zanaty F, WayA. Egypt Demographic and Health Survey 2014. Cairo, Egypt: Ministry of Health, El-Zanaty and Associates, and Macro International; 2015.

5. Gros A, Roussel M, Sauvadet E, et al. The sensitivity of neutrophil CD64 expression as a biomarker of bacterial infection is low in critically ill patients. Intensive Care Med. 2012;38(3):445-452.

6. Hassan U, Ghonge T, Reddy Jr. B, et al. A point-of-care microfluidic biochip for quantification of CD64 expression from whole blood for sepsis stratification. Nat Commun. 2017;8(8):15949. 
7. Sheneef A, Mohamed T, Boraey NF, Mohammed MA. Neutrophil CD11b, CD64 and lipocalin-2: early diagnostic markers of neonatal sepsis. Egypt J Immunol. 2017;24(1):29-36.

8. Mussap M, Noto A, Fravega M, Fanos V. Soluble CD14 subtype presepsin (sCD14-ST) and lipopolysaccharide binding protein (LBP) in neonatal sepsis: new clinical and analytical perspectives for two old biomarkers. J Matern Fetal Neonatal Med. 2011;24(Suppl 2):12-14.

9. Gibot S, Cravoisy A, Levy B, Bene MC, Faure G, Bollaert PE. Soluble triggering receptor expressed on myeloid cells and the diagnosis of pneumonia. N Engl J Med. 2004;350(5):451-458.

10. Palazzo SJ, Simpson TA, Simmons JM, Schnapp LM. Soluble triggering receptor expressed on myeloid cells-1 (sTREM-1) as a diagnostic marker of ventilator-associated pneumonia. Respir Care. 2012;57(12):2052-2058.

11. Ye W, Hu Y, Zhang R, Ying K. Diagnostic value of the soluble triggering receptor expressed on myeloid cells-1 in lower respiratory tract infections: a meta-analysis. Respirology. 2014;19(4):501-507.

12. Ballard JL, Khoury JC, Wedig K, Wang L, Eilers-Walsman BL, Lipp R. New Ballard score, expanded to include extremely premature infants. J Pediatr. 1991;119(3):417-423.

13. Töllner U. Early diagnosis of septicemia in the newborn. Eur J Pediatr. 1982;138(4):331-337.

14. Medhat $\mathrm{H}$, Khashana A, El Kalioby M. Incidence of neonatal infection in South Sinai, Egypt Int J Infect. 2017;4(1):e36615.

15. Bhandari V. Effective biomarkers for diagnosis of neonatal sepsis. J Pediatric Infect Dis Soc. 2014;3(3):234-245.

16. Khair KB, Rahman MA, Sultana T. Role of hematologic scoring system in early diagnosis of neonatal septicemia. Bangabandhu Sheikh Mujib Medical University Journal. 2010;3(2):62-67.

17. Paul D, IbneSattar A, Roy C. Role of neutrophil CD64 as an emerging biomarker in the evaluation of neonatal sepsis. Int Clin Pathol J. 2017;4(5):114-118.

18. Ng PC, Lam HS. Diagnostic markers for neonatal sepsis. Curr Opin Pediatr. 2006;18(2):125-131.

19. du J, Li L, Dou Y, Li P, Chen R, Liu H. Diagnostic utility of neutrophil CD64 as a marker for early-onset sepsis in preterm neonates. PLoS One. 2014;9(7):e102647.

20. de Benedetti F, Auriti C, D'Urbano LE, et al. Low serum levels of mannose binding lectin are a risk factor for neonatal sepsis. Pediatr Res. 2007;61(3):325-328.

21. Dzwonek AB, Neth OW, Thiébaut R, et al. The role of mannose-binding lectin in susceptibility to infection in preterm neonates. Pediatr Res. 2008;63(6):680-685.

22. Osman AS, Awadallah MG, Tabl HAE-M, Abed NT, Goudah ES. Presepsin as a novel diagnostic marker in neonatal septicemia. Egyptian J Med Microbiol. 2015;24(3):21-26.

23. Małgorzata S, Behrendt J, Szymańska A. Diagnostic value of presepsin (sCD14-ST subtype) evaluation in the detection of severe neonatal infections. Int J Res Studies in Biosciences. 2015;3:110-116.

24. Topcuoglu S, Arslanbuga C, Gursoy T, et al. Role of presepsin in the diagnosis of late-onset neonatal sepsis in preterm infants. $J$ Matern Fetal Neonatal Med. 2016;29(11):1834-1839.
25. Mussap M, Puxeddu E, Puddu M, et al. Soluble CD14 subtype (sCD14ST) presepsin in premature and full term critically ill newborns with sepsis and SIRS. Clinica Chimica Acta. 2015;451:65-70.

26. Ozdemir AA, Elgormus Y. Diagnostic value of presepsin in detection of early-onset neonatal sepsis. Am J Perinatol. 2017;34(6): $550-556$.

27. Adly AA, Ismail EA, Andrawes NG, El-Saadany MA. Circulating soluble triggering receptor expressed on myeloid cells-1 (sTREM-1) as diagnostic and prognostic marker in neonatal sepsis. Cytokine. 2014;65(2):184-191.

28. Saldir M, Tunc T, Cekmez F, et al. Endocan and soluble triggering receptor expressed on myeloid cells-1 as novel markers for neonatal sepsis. Pediatr Neonatol. 2015;56(6):415-421.

29. Zeitoun AA, Gad SS, Attia FM, Abu Maziad AS, Bell EF. Evaluation of neutrophilic CD64, interleukin 10 and procalcitonin as diagnostic markers of early- and late-onset neonatal sepsis. Scand J Infect Dis. 2010;42(4):299-305.

30. Dilli D, Oğuz ȘS, Dilmen U, Köker MY, Kızılgün M. Predictive values of neutrophil CD64 expression compared with interleukin-6 and C-reactive protein in early diagnosis of neonatal sepsis. J Clin Lab Anal. 2010;24(6):363-370.

31. Morsy AA, Elshall LY, Zaher MM, Abd Elsalam M, Nassr AE. CD64 cell surface expression on neutrophils for diagnosis of neonatal sepsis. Egypt J Immunol. 2008;15(2):53-61.

32. Soni S, Wadhwa N, Kumar R, et al. Evaluation of CD64 expression on neutrophils as an early indicator of neonatal sepsis. Pediatr Infect Dis $J$. 2013;32(1):e33-e37.

33. Bassuoni MA, Donia M, Helwa MA. Neutrophil expression of CD64 in the diagnosis of early-onset neonatal sepsis. Menoufia Med J. 2014;27(2):284-289.

34. Jia LQ, Shen YC, Hu QJ, et al. Diagnostic accuracy of neutrophil CD64 expression in neonatal infection: a meta-analysis. J Int Med Res. 2013;41(4):934-943.

35. Zou Q, Wen W, Zhang XC. Presepsin as a novel sepsis biomarker. World J Emerg Med. 2014;5(1):16.

36. Endo S, Suzuki Y, Takahashi G, et al. Usefulness of presepsin in the diagnosis of sepsis in a multicenter prospective study. $J$ Infect Chemother. 2012;18(6):891-897.

37. Shi J, Tang J, Chen D. Meta-analysis of diagnostic accuracy of neutrophil CD64 for neonatal sepsis. Ital J Pediatr. 2016;42(1):57.

38. Mazzucchelli I, Garofoli F, Ciardelli L, et al. Diagnostic performance of triggering receptor expressed on myeloid cells-1 and CD64 index as markers of sepsis in preterm newborns. Pediatr Crit Care Med. 2013;14(2):178-182.

39. Sabry J, Elfeky O, Elsadek A, Eldaly A, Resident of Pediatrics, Ministry of Health, Egypt. Presepsin as an early reliable diagnostic and prognostic marker of neonatal sepsis. Int J Adv Res. 2016;4(6): $1538-1549$.

40. Sarode R, Ingole N, Jasani B, Nataraj G, Nanavati R, Mehta P. Role of CD64 in the diagnosis of neonatal sepsis. Int J Contemp Med Res. 2014;4(9):1959-1963. 


\section{Supplementary materials}

Table SI General and clinico-laboratory data of EOS and LOS neonates under study

\begin{tabular}{|c|c|c|c|c|}
\hline $\begin{array}{l}\text { Studied groups } \\
\text { Items }\end{array}$ & Early onset sepsis $(n=17)$ & Late onset sepsis $(n=13)$ & Test of significance & $P$-value \\
\hline \multicolumn{5}{|l|}{ Sex } \\
\hline Boys & $13(76.5 \%)$ & $8(61.5 \%)$ & & \\
\hline Girls & $4(23.5 \%)$ & $5(38.5 \%)$ & $\chi^{2}=0.6$ & 0.4 \\
\hline Gestational age (weeks) $($ mean \pm SD) & $37.8 \pm 1.7$ & $37.9 \pm 1.8$ & $t$-test $=0.7$ & 0.8 \\
\hline Birth weight $(\mathrm{g})($ mean $\pm \mathrm{SD})$ & $3 \pm 0.9$ & $2.8 \pm 0.3$ & $t$-test $=0.5$ & 0.7 \\
\hline Length $(\mathrm{cm})($ mean $\pm \mathrm{SD})$ & $48.5 \pm 2$ & $47.3 \pm 1.9$ & $t$-test $=0.6$ & 0.2 \\
\hline Head circumference $(\mathrm{cm})($ mean $\pm \mathrm{SD})$ & $33.8 \pm 1.3$ & $29.3 \pm 10$ & $t$-test $=0.7$ & 0.3 \\
\hline WBCs $\left(10^{3} / \mu \mathrm{L}\right)($ mean $\pm S D)$ & $17.3 \pm 5.6$ & $22.2 \pm 9.8$ & $t$-test $=0.8$ & 0.4 \\
\hline $\mathrm{Hb}(\mathrm{g} / \mathrm{dL})($ mean $\pm \mathrm{SD})$ & $13.2 \pm 2.4$ & $12.3 \pm 1.7$ & $t$-test $=1.1$ & 0.5 \\
\hline HCT (\%) (mean \pm SD) & $38.8 \pm 5.8$ & $37.6 \pm 6.9$ & $t$-test $=0.7$ & 0.2 \\
\hline Platelets $\left(10^{3} / \mu \mathrm{L}\right)($ mean $\pm \mathrm{SD})$ & $102.3 \pm 6 \mid$ & $182.5 \pm 83.8$ & $t$-test $=3.5$ & $0.002 *$ \\
\hline Apgar score at 5 minutes (mean \pm SD) & $7.4+0.8$ & $8+0.8$ & $t$-test $=0.8$ & 0.3 \\
\hline Tollner score (mean \pm SD) & $12.4+3.9$ & $12.9+2.8$ & $t$-test $=0.7$ & 0.4 \\
\hline Hematological score (mean \pm SD) & $4.7+0.7$ & $4.9+0.7$ & $t$-test $=0.7$ & 0.6 \\
\hline
\end{tabular}

Note: $* P \leq 0.05$ is significant.

Abbreviations: $\mathrm{Hb}$, hemoglobin; $\mathrm{HCT}$, hematocrit; WBC, white blood cell.

Table S2 Levels of CRP, presepsin, nCD64, and sTREM-I in EOS and LOS studied group

\begin{tabular}{|l|l|l|l|l|}
\hline $\begin{array}{l}\text { Studied groups } \\
\text { Items }\end{array}$ & $\begin{array}{l}\text { CRP }(\mathbf{m g} / \mathbf{L}) \\
\text { (mean } \pm \text { SD) }\end{array}$ & $\begin{array}{l}\text { Presepsin } \mathbf{( p g / m L )} \\
\text { (mean } \pm \text { SD) }\end{array}$ & $\begin{array}{l}\text { nCD64 (MFI) } \\
\text { (mean } \pm \text { SD) }\end{array}$ & $\begin{array}{l}\text { sTREM-I (ng/L) } \\
\text { (mean } \pm \text { SD) }\end{array}$ \\
\hline EOS & $30.4 \pm I 8.8$ & $3,442 \pm I, 094$ & $1 I 8 \pm 7.4$ & $263.6 \pm 100$ \\
LOS & $26.8 \pm 16.3$ & $3,345.5 \pm I, 244$ & $92.2 \pm 5.6$ & $230 \pm 129$ \\
\cline { 2 - 5 } -value & $P I=0.500$ & $P I=0.784$ & $P I=0.137$ & $P I=0.075$ \\
& $P 2=0.00 I^{*}$ & $P 2=0.00 I^{*}$ & $P 2=0.002^{*}$ & $P 2=0.00 I^{*}$ \\
& $P 3=0.002^{*}$ & $P 3=0.00 I^{*}$ & $P 3=0.00 I^{*}$ & $P 3=0.002^{*}$ \\
\hline
\end{tabular}

Notes: $P$ I, comparison between EOS and LOS; P2, comparison between control group and EOS; P3, comparison between control group and LOS using independent $t$-test. $* P \leq 0.05$ is significant.

Abbreviations: EOS, early onset sepsis; LOS, late onset sepsis.

Table S3 Levels of CRP, presepsin, nCD64, and sTREM-I in proven sepsis and probable sepsis groups

\begin{tabular}{|l|l|l|l|l|}
\hline $\begin{array}{l}\text { Studied groups } \\
\text { Items }\end{array}$ & $\begin{array}{l}\text { CRP (mg/L) } \\
\text { (mean } \pm \text { SD) }\end{array}$ & $\begin{array}{l}\text { Presepsin (pg/mL) } \\
\text { (mean } \pm \text { SD) }\end{array}$ & $\begin{array}{l}\text { nCD64 (MFI) } \\
\text { (mean } \pm \text { SD) }\end{array}$ & $\begin{array}{l}\text { sTREM-I (ng/L) } \\
\text { (mean } \pm \text { SD) }\end{array}$ \\
\hline Proven sepsis & $30 \pm I 8.8$ & $3453 \pm I, 094$ & $90.8 \pm 7.4$ & $130 \pm I 00$ \\
Probable sepsis & $\mid 8 \pm 8.3$ & $2864 \pm I, 244$ & $\mid 22 \pm 5.6$ & $\mid 79 \pm I 29$ \\
\cline { 2 - 5 } -value & $P I=0.04^{*}$ & $P I=0.6$ & $P I=0.3$ & $P I=0.4$ \\
& $P 2=0.00 I^{*}$ & $P 2=0.00 I^{*}$ & $P 2=0.00 I^{*}$ & $P 2=0.00 I^{*}$ \\
& $P 3=0.334$ & $P 3=0.00 I^{*}$ & $P 3=0.00 I^{*}$ & $P .00 I^{*}$ \\
\hline
\end{tabular}

Notes: $P I$, comparison between proven and probable sepsis; $P 2, P 3$, comparison of control group separately with proven and probable sepsis, respectively, using independent $t$-test. $* P \leq 0.05$ is significant.

resistance and the mechanisms of resistance development and diffusion in both hospitals and the community. The manuscript management system is completely online and includes a very quick and fair peerreview system, which is all easy to use. Visit http://www.dovepress.com/ testimonials.php to read real quotes from published authors. 